\title{
Clinical, biochemical and metabolic characterization of patients with short- chain enoyl-CoA hydratase(ECHS1) deficiency: two case reports and the review of the literature
}

Hua Yang ${ }^{1,2}$ and Dan $Y u^{1,2^{*}}$

\begin{abstract}
Background: Short-chain enoyl-CoA hydratase (SCEH or ECHS1) deficiency is a rare congenital metabolic disorder caused by biallelic mutations in the ECHS gene. Clinical phenotype includes severe developmental delay, regression, dystonia, seizures, elevated lactate, and brain MRI abnormalities consistent with Leigh syndrome (LS). SCEH is most notably involved in valine catabolism. There is no effective treatment for the disease, patients may respond to dietary restriction of valine and supplementation of N-acetylcysteine

Case presentation: We describe two patients who presented in infancy or early childhood with SCEH deficiency. Both patients were shown to harbor heterozygous or homozygous variants in the ECHS1 gene, and developmental retardation or regression as the onset manifestation. Brain MRI showed abnormal signals of bilateral pallidus. Urine metabolic examination showed increased levels of 2,3-dihydroxy-2-methylbutyric acid and S-(2-carboxypropyl) cysteamine S-(2-carboxypropoxypropyl) cysteamine (SCPCM). A valine restricted diet and combined of Nacetylcysteine supplementation were utilized in the two patients.

Conclusions: In clinical practice, The elevated urinary 2,3-dihydroxy-2-methylbutyrate, S-(2-carboxypropyl) cysteine, $\mathrm{S}$-(2-carboxypropyl) cysteine and N-acetyl-S-(2-carboxypropyl) cysteine levels might be clues for diagnosis of SCEH deficiency which can be confirmed throughGenetic sequencing of ECHS1 gene. Early cocktail therapy, valine restrictied diet and $\mathrm{N}$-acetylcysteine supplementation could improve the prognosis of patients.
\end{abstract}

Keywords: Short-chain enoyl-CoA hydratase deficiency, ECHS1, urinary metabolic profile

\section{Background}

Short-chain enoyl-CoA hydratase(SCEH or ECHS1) deficiency, also known as crotonase deficiency, is a rare autosomal recessive hereditary disease (OMIM 616277). It was first reported by Peters et al. (2014)and caused by pathogenic variants in the ECHS1 gene [1]. Clinical manifestations include mental retardation or degeneration, dystonia, seizure, hyperlacticemia, elevated lactate, and brain MRI abnormalities consistent with Leigh

\footnotetext{
* Correspondence: yd540@126.com

'Department of Pediatrics, West China Second University Hospital, Ren Min South Road 3rd Second 20\#, Chengdu 610041, Sichuan, China

${ }^{2}$ Key Laboratory of Obstetric \& Gynecologic, Pediatric Diseases and Birth Defects of Ministry of Education, Chengdu, China
}

syndrome (LS). Theincidence of SCEH deficiency is very low, and there are only 44 cases reported abroad (Table 1) and at present, no SCEH deficiency has been reported in China. In this study, the clinical data of two patients with SCEH deficiency were reported, and the relevant literatures were reviewed to improve clinicians' understanding of this disease.

\section{Case presentation \\ Case 1}

The child was a 2 year- old girl. She was born by caesarian section for fetal transverse presentation at term with birth weight $3.25 \mathrm{~kg}$ (percentile 50th-75th), height 48

(c) The Author(s). 2020 Open Access This article is distributed under the terms of the Creative Commons Attribution 4.0 International License (http://creativecommons.org/licenses/by/4.0/), which permits unrestricted use, distribution, and reproduction in any medium, provided you give appropriate credit to the original author(s) and the source, provide a link to the Creative Commons license, and indicate if changes were made. The Creative Commons Public Domain Dedication waiver (http://creativecommons.org/publicdomain/zero/1.0/) applies to the data made available in this article, unless otherwise stated. 


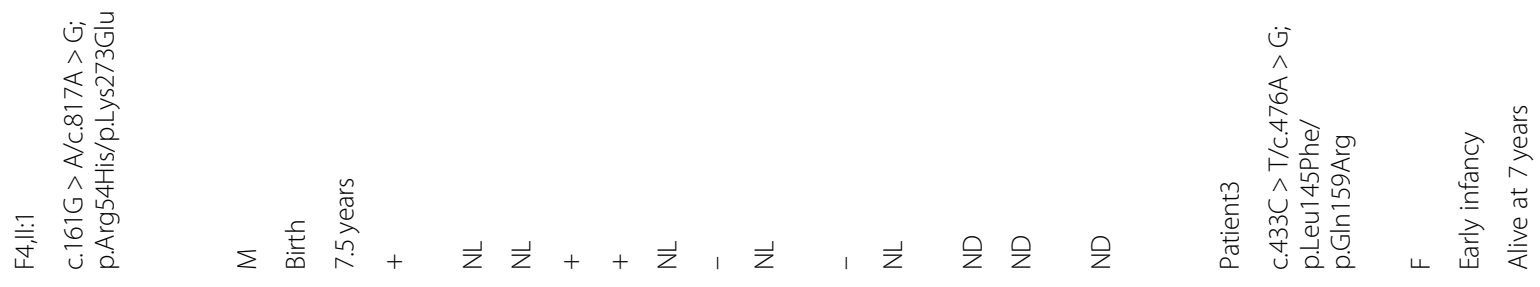

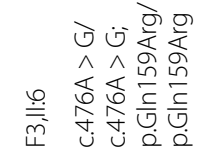

\section{के 인}

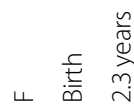

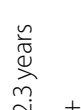

$\bar{z}++\bar{z}+$

ฉ

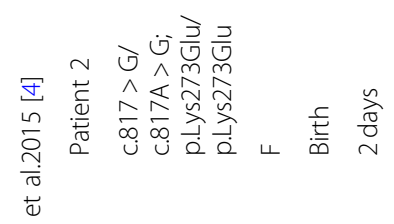

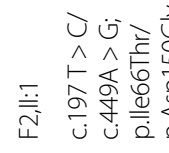

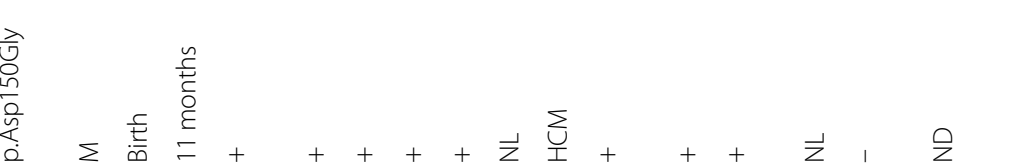

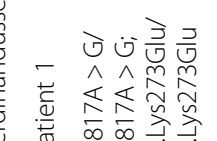

$\leftarrow \stackrel{\frac{c}{E}}{\frac{5}{\oplus}}$

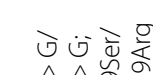

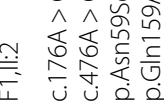

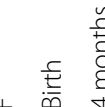

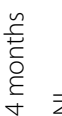

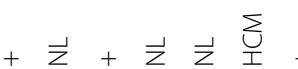

$\bar{z}+$

z

そ

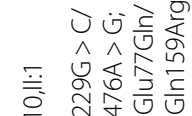

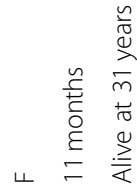

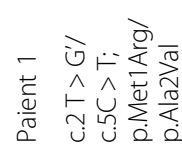

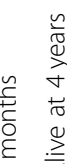

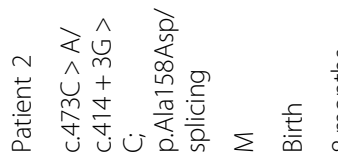

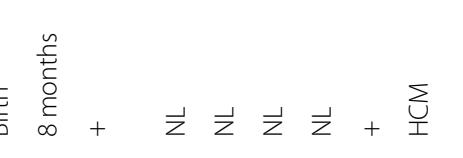

$\frac{1}{z}+$

$\vec{z} z \quad \gtrless$

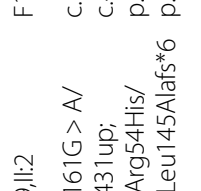

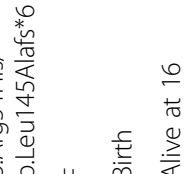

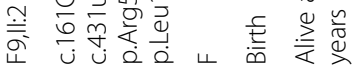

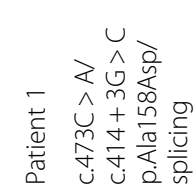

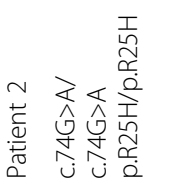

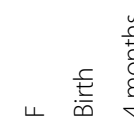

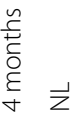

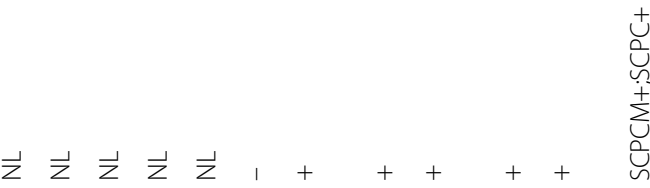

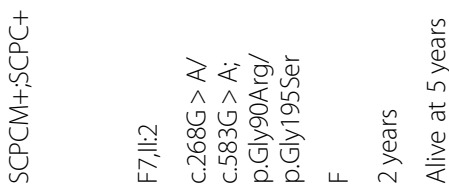

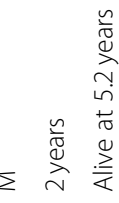<smiles>[CH]1[CH]C1</smiles>

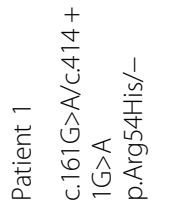

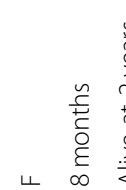

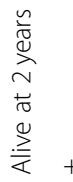

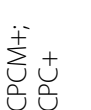

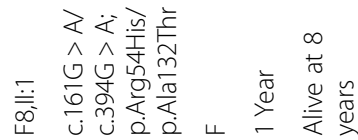

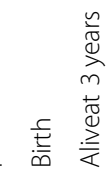




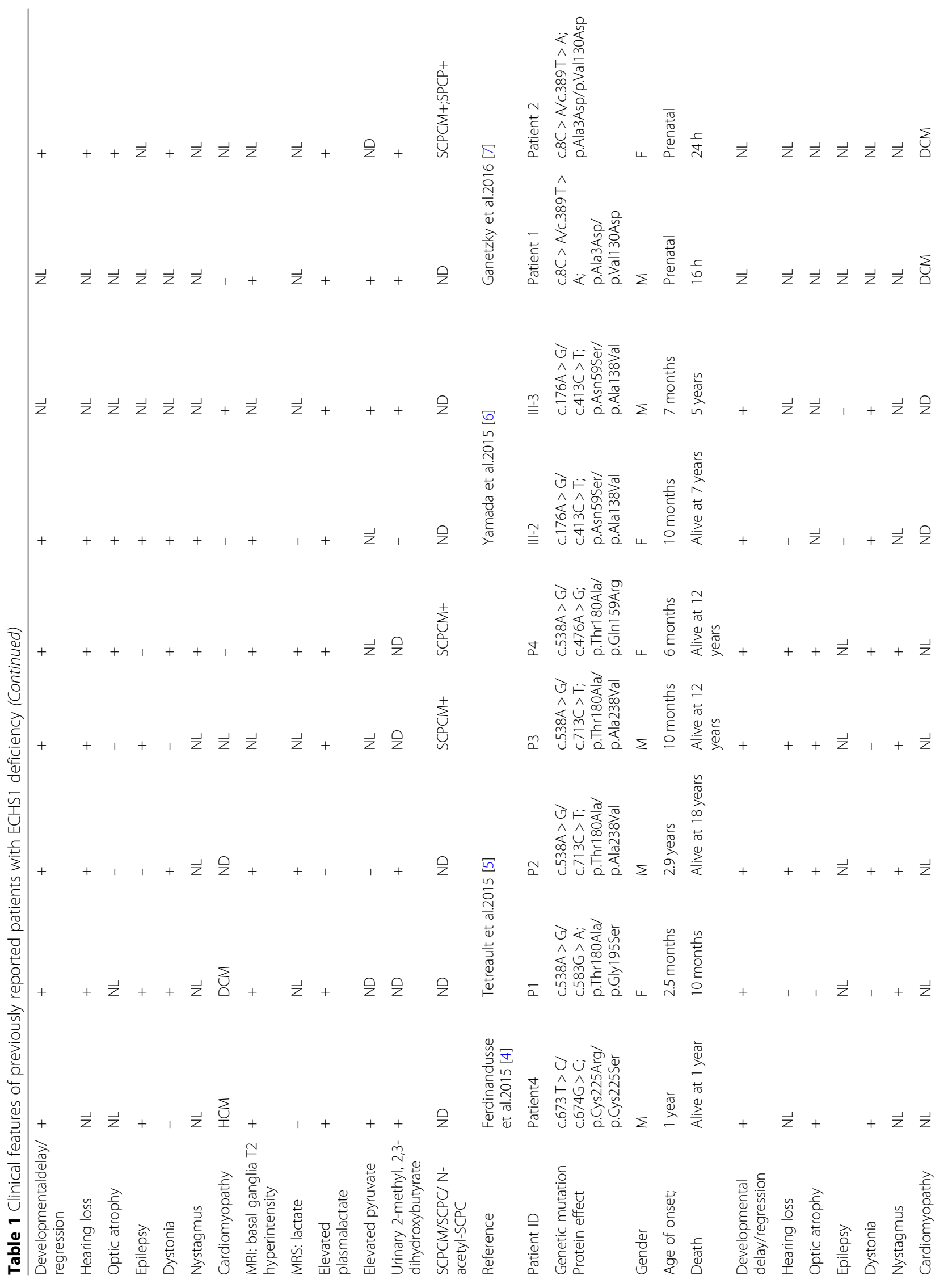




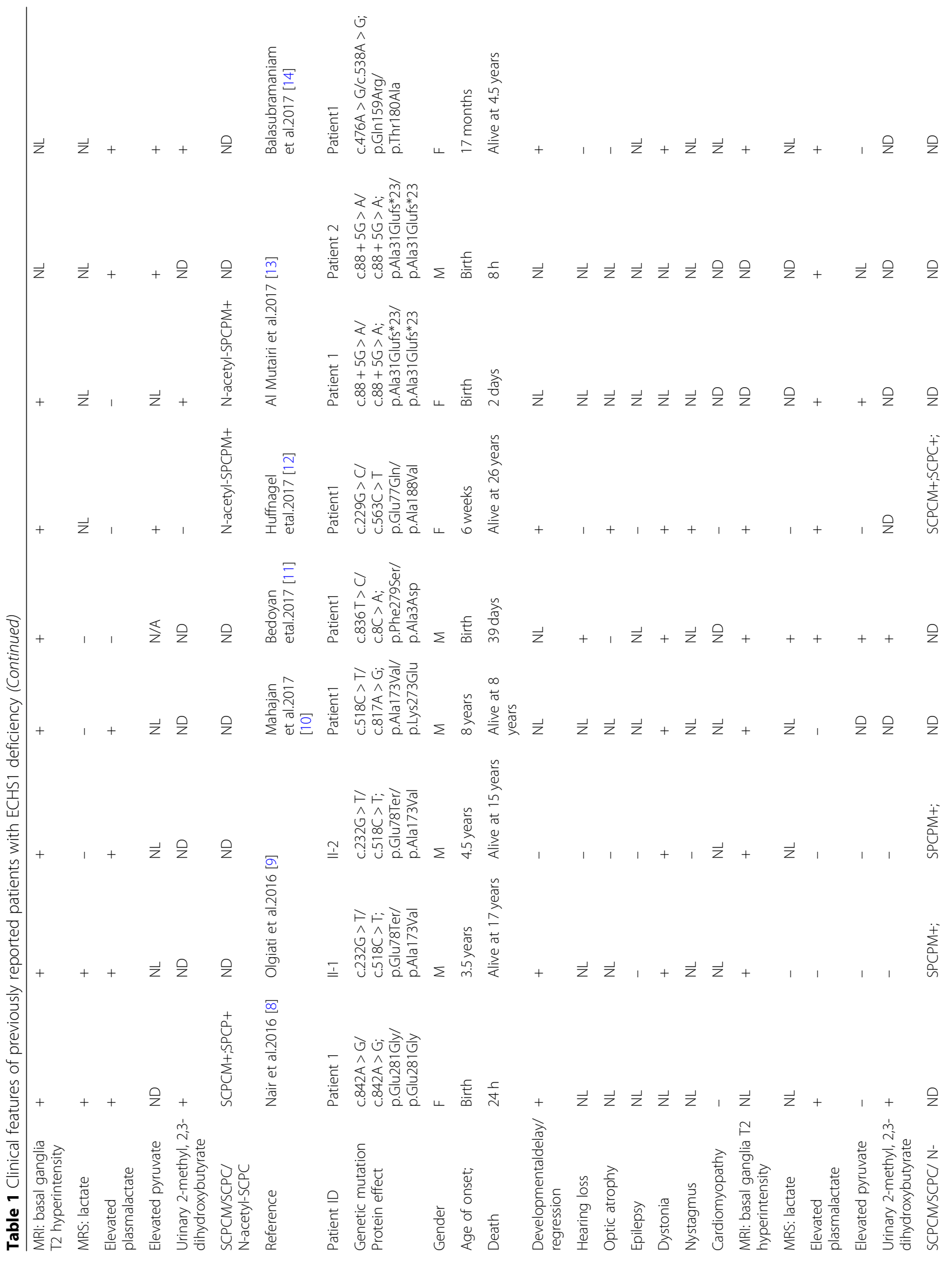




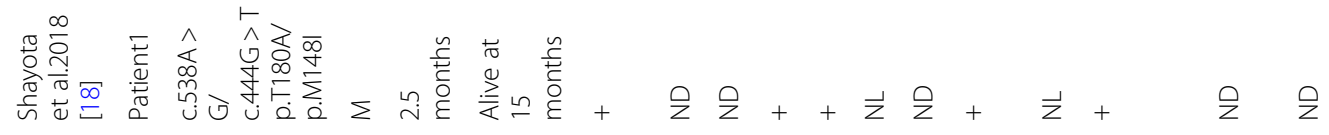

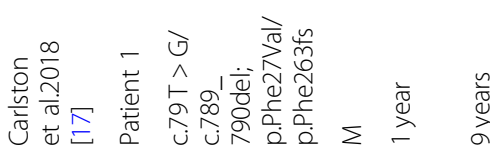

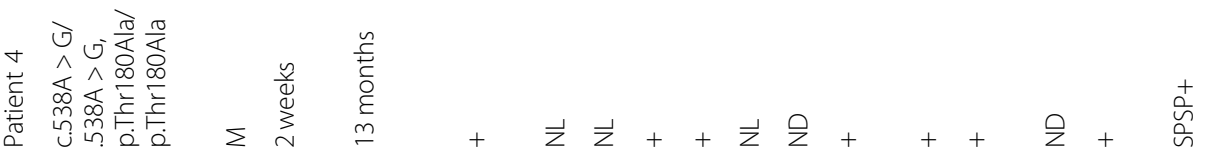

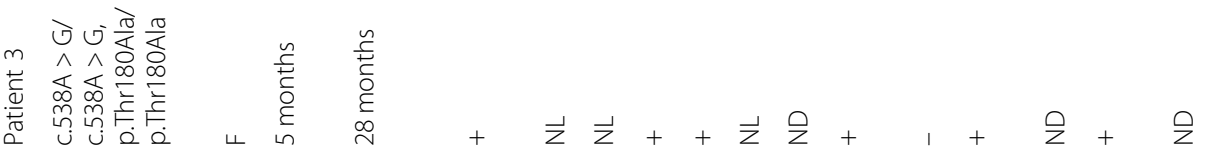

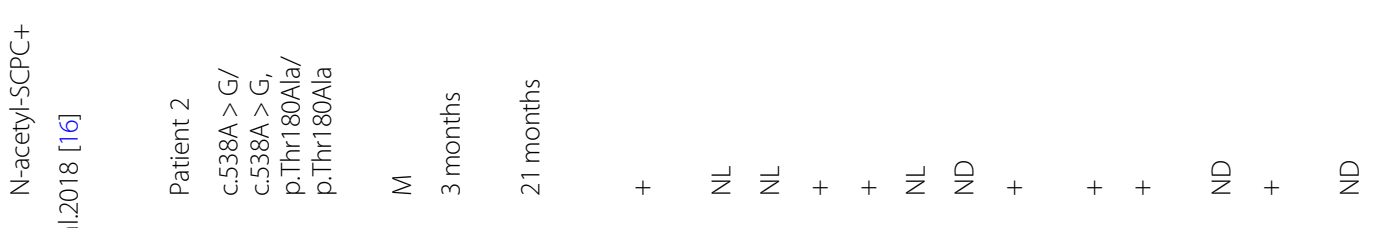

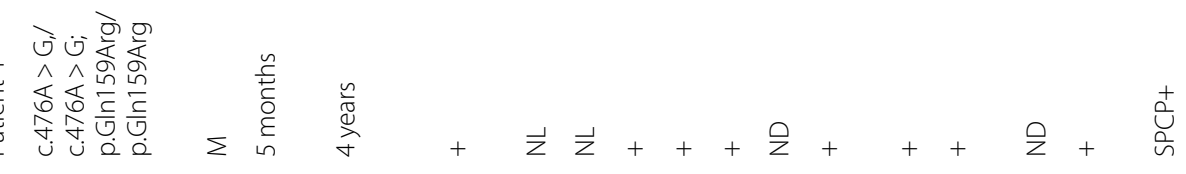

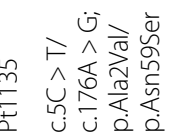

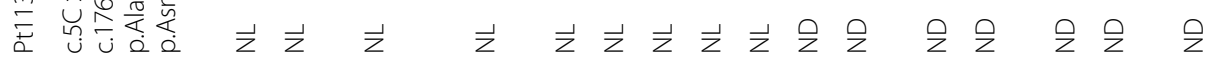

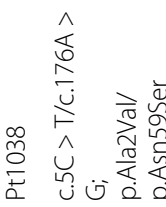

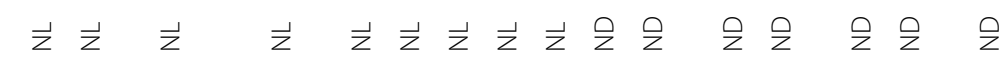
$\stackrel{\dot{0}}{\wedge}$

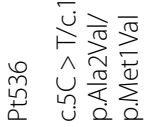
$\vec{z} \vec{z} \quad \vec{z} \vec{z} \vec{z} \vec{z} \stackrel{\mathrm{z}}{\mathrm{z}} \mathrm{z} \stackrel{\mathrm{z}}{\mathrm{z}}$

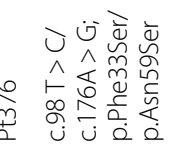


cm (percentile 10th-25th), and head circumference 34 $\mathrm{cm}$ (percentile 50th). Her birth history was unremarkable and her early milestones were appropriate. From 8 months of age onwards motor developmental delay was noted. By 1 year of age, she had obvious developmental regression due to infection, but symptoms improved slightly after the infection was treated. The patient was first evaluated in our hospital at 14 months due to severe developmental delay. She was unable to sit alone or crawl, accompanied by language dysfunction, poor fine motion, and taking simple instructions. At this time, the patient growth parameters were: weight of $10 \mathrm{~kg}$ (percentile 3th-10th), length of $83 \mathrm{~cm}$ (percentile 10th25th), and head circumference of $46.5 \mathrm{~cm}$ (percentile 25th-50th). Developmentally, she had hypotonia, language development delay, mental retardation and nystagmus. Elevated levels of blood lactate $4.84 \mathrm{mmol} / \mathrm{L}$ (normal $\quad 0.7-2.1 \mathrm{mmol} / \mathrm{L}$ ), pyruvic acid $122 \mu \mathrm{mol} / \mathrm{L}$ (normal 20-100 mmol/L), and TSH3UL1.081 $\mathrm{mTU} /$ L(normal 1.7-9.1 mTU/L)were noted. Blood ammonia, blood routine, $\beta$-hydroxybutyric acid, ceruloplasmin, blood calcium/phosphorus/alkaline phosphatase, serum vitamin D, liver and kidney function, electrolytes were all normal. Urine organic acid analysis and electroencephalogram (EEG) in 2018 was normal, brain MRI (Fig. 1a, A2) showed bilateral pale globular morphology and signal changes which suggested the possibility of genetic metabolic diseases. MRS showed bilateral lesions with inverted lactate peaks. Re-examination of urine metabolism in 2019 showed that 2,3-dihydroxy-2-methylbutyric acid was detected significantly.

The genomic DNA was isolated from blood and processed for IDT The xGen Exome Research Panel on Hiseq-Illumina NGS platform. Whole exome sequencing analysis (Fig. 2) revealed that there were ECHS1 gene heterozygous mutations following: c.161G > A in exon 2
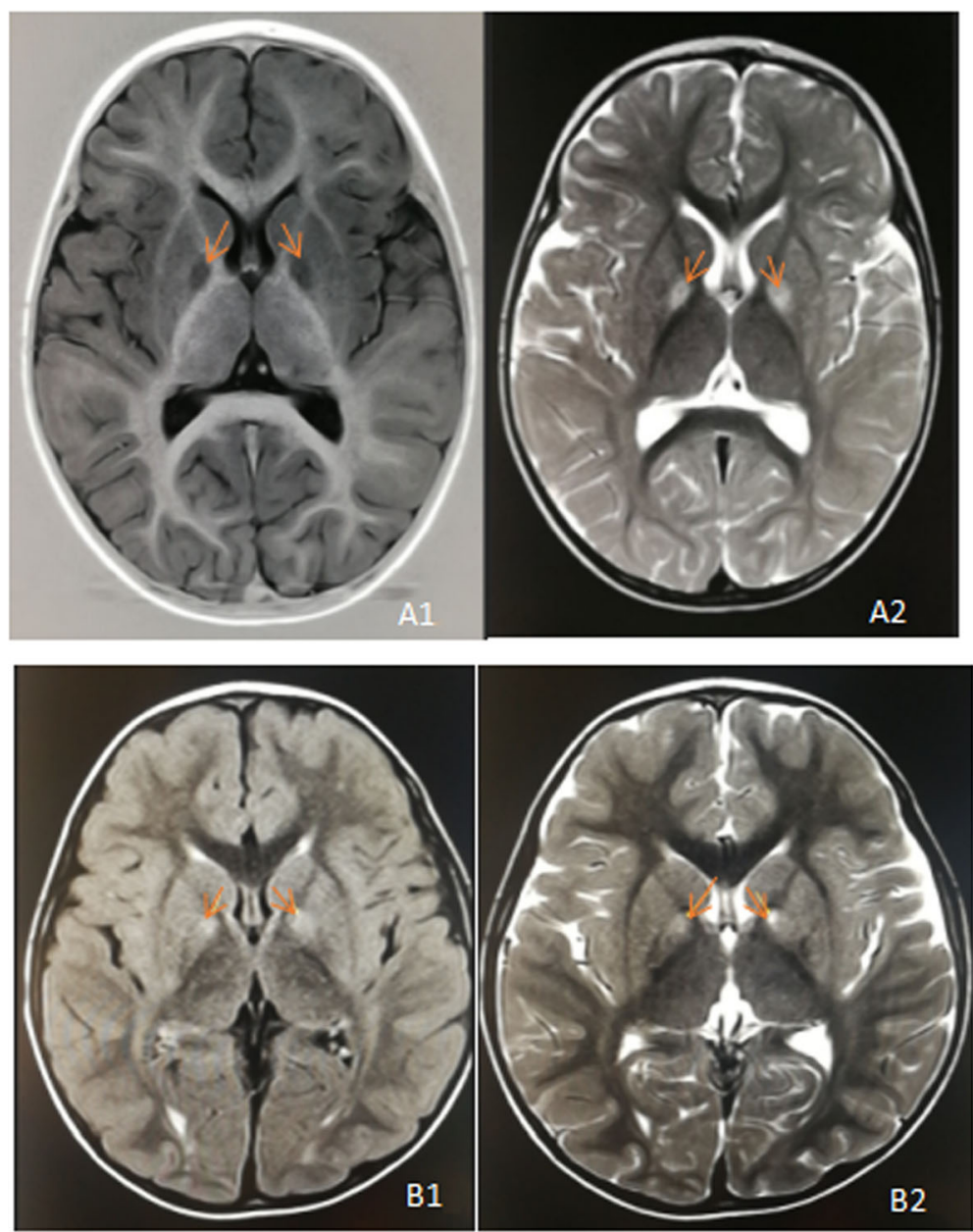

Fig. 1 a Brain MRI of patient 1 showed bilateral globus pallidus swelling and abnormal signal (as shown by arrow), T1 W1 (A1) showed low signal and T2 W1 (A2) showed high signal; b Brain MRI of patient 2 showed a little patchy abnormal signal of bilateral globus pallidus (as shown by arrow), small patchy shadow of left globus pallidus adjacent to anterior limb of internal capsule showed slightly obvious, and both T2WI-flair(B1) and T2WI(B2)showed high signal 


\section{c.161(exon2) G>A \\ Patient 1 \\ (Heterozygous type)}
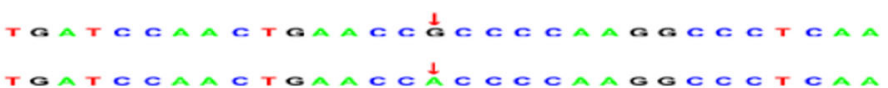

(Wild type)
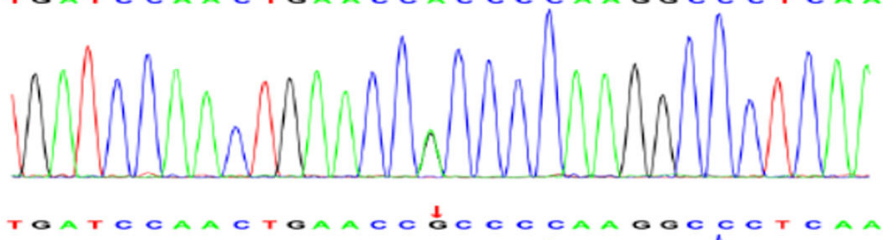

Father of patient1

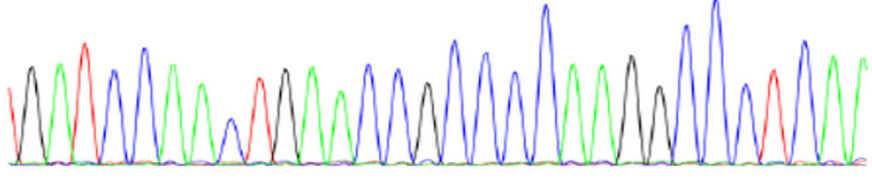

Mother of patient 1

(Heterozygous type)

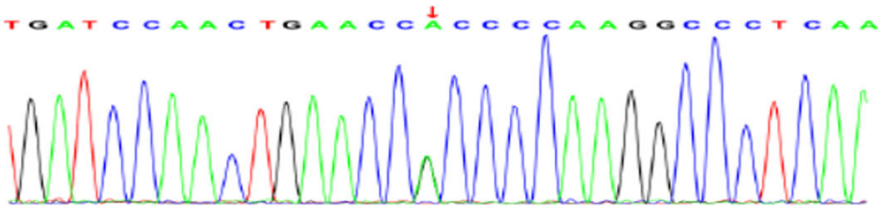

c. $414+1(\mathrm{IVS} 3) \mathrm{G}>\mathrm{A}$

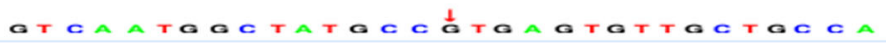

GTCAATGOCTATOCC ATGAOTOTTOCTOCGA

Patient 1

(Heterozygous type)

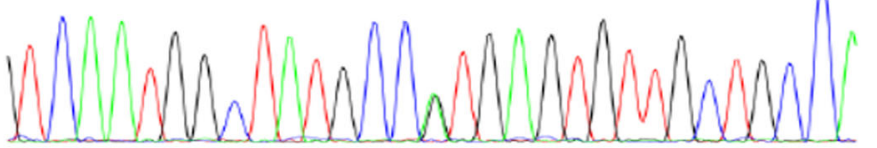

Father of patient1

(Heterozygous type)

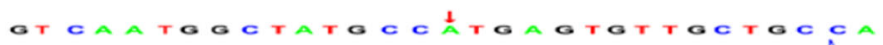

Mother of patient 1
(wild type)
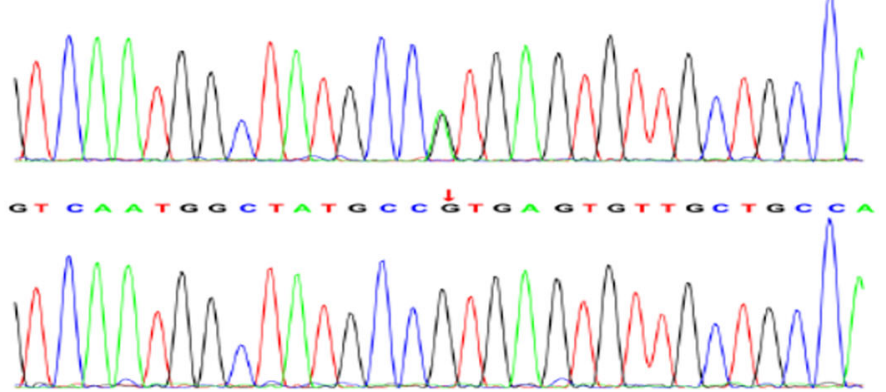

Fig. 2 Gene detection of patients 1 and their parents in total exome gene detection. The ECHS1 gene of patient 1 had c.161G > A and c.414 + $1 \mathrm{G}>$ A compound heterozygous mutation, the ECHS1 gene of his mother had c.161G > A heterozygous mutation, and the ECHS1 gene of his father had $\mathrm{C} .414+1 \mathrm{G}>\mathrm{A}$ heterozygous mutation

in the chr10:135184189 region and c.414 + $1 \mathrm{G}>\mathrm{A}$ exists in intron 3 in the chr10:135183407 region, which were inherited from her mother and father respectively. The c.161G > A (p.Arg54His) has been previously reported in compound heterozygous state in clinically affected patients (Haack et al., 2015), and which may be pathogenic. c. $414+1 \mathrm{G}>\mathrm{A}$ genes were not reported in ClinVar, OMIM and HGMD databases, and Mutation Taster suggested the variant to be disease-causing.

\section{Case 2}

Patient number 2 was a 5 year- old boy. He was born at 39 weeks of gestation by normal vaginal delivery after an uneventful pregnancy and the product of the second pregnancy of his mother (spontaneous abortion of the first fetus). His birth weight was $3.3 \mathrm{~kg}$ (percentile 25th50th), height $49 \mathrm{~cm}$ (percentile 10th-25th), head circumference $33.5 \mathrm{~cm}$ (percentile 25th-50th) with normal Apgar scores. There were no metabolic disturbances observed post-delivery. Developmental milestones were normally reached. Unsteady gait was noticed at 2 years of age with developmental regression, foot-pad walking, unable to run and jump, involuntary movement, poor fine activity, hypermyotonia and language dysfunction. His growth parameters were a weight of $11.5 \mathrm{~kg}$ (percentile 10th-25th), length of $85 \mathrm{~cm}$ (percentile 10th- 
25th), and head circumference of $48 \mathrm{~cm}$ (percentile 25th-50th). Elevated levels of $\alpha$-hydroxybutyrate dehydrogenase $223 \mathrm{U} / \mathrm{L}$ (normal 2-25 U/L), lactate dehydrogenase $266 \mathrm{U} / \mathrm{L}$ (normal 106-211 U/L), creatine kinase isoenzyme $36 \mathrm{U} / \mathrm{L}$ (normal 0-25 U/L) were noted. Blood lactic acid, blood ammonia, pyruvic acid, blood routine, blood gas analysis, liver and kidney function, electrolytes were all normal. No abnormalities were found in the serum tandem mass spectrometry and urinary organic acid analysis in2017. Brian MRI (Fig. 1B1, B2) showed a few abnormal signals in the bilateral globus pallidus, and slightly obvious shadows in the left globus pallidus. Meanwhile, EEG was normal. Re-screening of urinary metabolism in 2019 suggested that 2, 3-dihydroxy-2methylbutyric acid was slightly increased. After conducting further special metabolic examination of the urine, it was found that the concentration of S-(2-caboxypropyl) cysteamine (SCPCM) was $2.83 \mathrm{micromol} / \mathrm{mmolCr}$, which is about four times the upper limit of reference value.

Detection of whole exome sequencing of genomic DNA showed that there was a c.74G > A mutation in the chr10: 135186764 region (exon 1) of ECHS1 gene. Family sequencing data showed that his mother carried the abovementioned heterozygous mutation. High-throughput sequencing data indicated that there was a deletion of heterozygosity in his father. c.74G > A was not reported in ClinVar, OMIM and HGMD, ExAC, dpSNP and other databases, and the missense mutation may be pathogenic.

\section{Discussion and conclusion}

SCEH(also known as crotonase) is a 290 amino acid protein encoded by ECHS1 on chromosome 10 and localized in the mitochondrial matrix as a $160 \mathrm{kD}$ ahomohexameric enzyme [4, 19]. SCEH has broad substrate specificity for acyl-CoAs with chain lengths up to 10 carbon atoms, although it shows greatest activity towards crotonyl-CoA. In the metabolic pathway, the functions of SCEH is to hydrate the double bond between the second and third carbon of enoyl-CoAs. In addition, its function in the oxidation of short chain acyl-CoAs, SCEH also has activity on the degradation intermediates of branched-chain acylCoAs, particularly valine, isoleucine and leucine. However, recent functional studies have shown that $\mathrm{SCEH}$ is a crucial enzyme in valine metabolism, but not isoleucine metabolism, and has limited effect on mitochondrial fatty acid oxidation $[11,13]$.

Pathogenic variants of biallelic allele in ECHS1 leads to the decrease of SCEH activity,and results in clinical manifestations of Leigh syndrome with early onset of hypotension, growth retardation or degeneration, cardiomyopathy, dystonia, sensorineural hearing loss, hypotension and malnutrition. Blood and cerebrospinal fluid (CSF) lactate and pyruvate levels are usually elevated. Brain MRI may show white matter changes or Leigh syndrome-like pattern affecting brainstem and basal ganglia, similar to other genetic energy metabolic disorders $[3,11,18]$. Both two patients we reported in this study had developmental retardation, dystonia, feeding difficulties and malnutrition. Patient 1 also had nystagmus, elevated blood lactate and pyruvate. Serum alpha-hydroxybutyrate dehydrogenase and lactate dehydrogenase and creatine kinase isoenzymes in patient 2 were elevated. Both two patients had abnormal signals of bilateral globus pallidus on brian MRI. Patient 1 showed inverted lactate peaks in bilateral lesions on brian MRS, which was consistent with the results reported in the literatures.

The pathophysiology of this disease is still unclear. It has been hypothesized to be secondary to toxic accumulation of intermediate metabolites that exert brain toxicity and derange mitochondrial energy production. Previous studies have shown that ECHS1 mutation can induce accumulation of active metabolites of methacrylyl-CoA and acryloyl-CoA [7, 13]. SCEH protein has high specificity and activity to methacrylyl-CoA, which is a toxic compound that tends to form adducts with cysteine and causes cell damage. Therefore, the loss of SCEH enzyme activity may lead to the accumulation of this toxic compound and pathogenicity [9].

Evidence is emerging to support that,levels may correlate with disease severity. In retrospective analysis of mitochondrial encephalopathy and transient 3-methylglutaconic aciduria caused by ECHS1 deficiency, Huffnagel et al. suggested that S-(2-carboxypropyl) cysteine, S-(2-carboxypropyl) cysteine and $\mathrm{N}$-acetyl-S-(2-carboxypropyl) cysteine might been useful diagnostic makers of SCEH deficiency [12]. For patients with mild forms of deficiency, only N-acetyl-S-(2-carboxypropyl) cysteine level is increased in the urine, suggesting that the metabolites of methacrylyl-CoA are important diagnostic markers of the mild and severe forms of ECHS1 deficiency. N-acetyl-S-(2-carboxypropyl)cysteine and its derivatives are formed from methacrylyl-CoA, so methacrylyl-CoA is also an important diagnostic marker for mild SCEHdeficiency $[6,12,20,21]$. Peter et al. found that the content of 2,3-dihydroxy-2-methylbutyrate in urine of two SCEHdeficiency patients increased in the study of ECHS1mutations in LS suggesting that the metabolite may be a common biochemical change in SCEHdeficiency [1].

Fitzsimons et al. studied the role of SCEH in the metabolism of fatty acids and branched chain amino acids in four patients with ECHS1 gene mutation. The results of enzyme activity measurement and western blot analysis strongly indicated that there was a correlation between residual SCEH activity and severity of clinical symptoms $[12,16]$. In this study, two patients received urine organic analysis, but no abnormality was found. The second urine metabolic test used urease method, and found 2, 3-dihydroxy-2-methylbutyric acid increased, which was related to the sensitivity of the detection method. 2,3-dihydroxy-2-methylbutyric acid in 
urine was only slightly elevated in patient 2 due to treatment. After further special urine examination was performed, S-(2-carboxypropyl) cysteamine was found increased significantly, which supported the diagnosis of SCEH deficiency and was consistent with gene diagnosis. The organic acid extraction technique in urine metabolism examination has low sensitivity in such patients and urease method can be used to improve the positive rate.

Three rare variants were detected by clinical exome sequencing of the probands.. Patient 1 had a compound heterozygous mutation of pathogenic variant c. $414+1 G>A$ and likely pathogenic variant c.161G $>$ A, which was inherited from her father and mother respectively. Patient 2 has a homozygous mutation of c.74G > A. His mother carried a heterozygous mutation and his father had a gene deletion. c.161G > A ECHS1 variant has been previously reported by Haack et al. for the first time in the study of mitochondrial encephalopathy with cardiac involvement caused by ECHS1 deficiency. In his study, three patients in 10 families were found to carry c.161G > A mutation gene. All these three patients and patient 1 in our study had onset in infancy, accompanied by developmental retardation. Some patients had the same manifestations as patient 1 , such as dystonia, nystagmus. Elevated serum lactate level, bilateral basal ganglia signal changes on brian MRI, and lactate peak on MRS were observed. Two of the three reported cases showed elevated urine S-(2-caboxypropyl)cysteamine concentration by urine specific metabolic screening. The c.161G > A variant has a population frequency of 0.000032 in East Asian. The pathogenic c.74G > A mutation is novel and never been reported before.

SCEH deficiency is a high mortality rate and poor prognosis disease. Most patients died of severe metabolic acidosis in infancy. So far, no standard therapy for ECHS1 deficiency exists for the treatment of SCEH deficiency. Some patients have received "cocktail therapy", and the production of the toxic methacrylyl-CoA and its derivatives might be avoided with specific dietary regimens that reduce the workload on the valine catabolic pathway [9]. Some researchers have observed the increase of $\mathrm{N}$-acetyl-S-(2-carboxypropyl) cysteine levels in patients with SCEH and $\mathrm{HIBCH}$, suggesting that glutathione metabolism may be the cause of sulfhydrylcontaining metabolites in urine. The combination of glutathione and glutathione S-transferase is an effective detoxification method for excretion of this abnormal metabolite through urine. Therefore, it is believed that supplementation of $\mathrm{N}$-acetylcysteine may be beneficial to increase the content of glutathione, thereby eliminating toxic metabolites [18]. Some experts suggest that the combined approach of $\mathrm{N}$-acetylcysteine supplementation and a restricted diet can be safely utilized in SCEH deficiency patients. In addition, there are literatures on ketogenic diet for SCEH deficiency [11, 16, 17], but the efficacy and safety of it remains yet to be studied. In this study, two patients were treated with cocktail therapy, low valine diet and rehabilitation therapy. Both of their motor functions were slightly improved after treatment.

In conclusion, SCEH deficiency caused by mutations of ECHS1 gene is a rare mitochondrial disease and patients typically present with psychomotor retardation, dystonia, nystagmus, cardiomyopathy, sensorineural hearing impairment, hyperlacticemia and Leigh syndrome-like cranial MRI in infants and young children. Elevated levels of 2,3dihydroxy-2-methylbutyrate, S-(2-carboxypropyl) cysteine, $\mathrm{S}$-(2-carboxypropyl) cysteine and N-acetyl-S-(2-carboxypropyl) cysteine can be diagnostic clues towards the disease spectrum of ECHS1 mutations in patients with an earlyonset and mitochondrial encephalopathy. Early cocktail therapy, valine restriction diet and $\mathrm{N}$-acetylcysteine supplementation can improve the prognosis of patients.

\section{Supplementary information}

Supplementary information accompanies this paper at https://doi.org/10. 1186/s12887-020-1947-z.

Additional file 1: CARE checklist.

Abbreviations

DCM: Dilated cardiomyopathy; HCM: Hypertrophic cardiomyopathy; N-acetylSCPC: N-acetyl-S-(2-carboxypropyl)-cysteine (N-acetyl-methacryl-I-cysteine; ND: Not determined; NL: Not listed; SCPC: S-(2-carboxypropyl)cysteine; SCPCM: S-(2-caboxypropyl)cysteamine

\section{Acknowledgements}

The authors thank the patients' parents for providing permission to use the information of their children, and also thank Dr. Mowshica Rajah for reviewing the manuscript.

\section{Authors' contributions}

HY and DY analyzed and interpreted the patient's data. HY wrote the draft of this article, DY revised this article, and Mowshica helped to ensure the English expression about the article more clear. All authors read and approved the final manuscript.

Funding

This work was supported by grants from the Science \& Technology Department of Sichuan Province (No. 2018SZ0123) for data collection and language polish

\section{Availability of data and materials}

All data generated or analysed during this study are included in this published article [and its Additional file 1].

Ethics approval and consent to participate

Informed consent was obtained from both sets of parents for routine and investigative studies.

Consent was obtained from the parents of two families for whole exome sequencing (WES) of two affected patients according to Ethics Committee of the West China Second University Hospital procedures.

\section{Consent for publication}

The study obtained informed written consent from the parents of the study participants in order to publish their clinical details and clinical images.

\section{Competing interests}

Each author declares that they have no competing interests, and certifies that he has no commercial association (e.g. consultancies, stock ownership, equity interest, patent/licensing arrangement, etc.) that might pose a conflict of interest in connection with the submitted article. 
Received: 21 August 2019 Accepted: 27 January 2020

Published online: 03 February 2020

\section{References}

1. Peters $H$, Buck N, Wanders $R$, et al. ECHS1 mutations in Leigh disease: a new inborn error of metabolism affecting valine metabolism. Brain. 2014;137(Pt 11):2903-8.

2. Sakai C, Yamaguchi S, Sasaki M, Miyamoto Y, Matsushima Y, Goto Y. ECHS1 mutations cause combined respiratory chain deficiency resulting in Leigh syndrome. Hum Mutat. 2014;36(2):232-9.

3. Haack TB, Jackson CB, Murayama K, et al. Deficiency of ECHS1 causes mitochondrial encephalopathy with cardiac involvement. Ann Clin Transl Neurol. 2015;2(5):492-509.

4. Ferdinandusse $\mathrm{S}$, Friederich MW, Burlina A, et al. Clinical and biochemical characterization of four patients with mutations in ECHS1. Orphanet J Rare Dis. 2015;10:79.

5. Tetreault M, Fahiminiya S, Antonicka $\mathrm{H}$, et al. Whole-exome sequencing identifies novel ECHS1 mutations in Leigh syndrome. Hum Genet. 2015; 134(9):981-91.

6. Yamada K, Aiba K, Kitaura Y, et al. Clinical, biochemical and metabolic characterisation of a mild form of human short-chain enoyl-CoA hydratase deficiency: significance of increased N-acetyl-S-(2-carboxypropyl)cysteine excretion. J Med Genet. 2015;52(10):691-8.

7. Ganetzky RD, Bloom K, Ahrens-Nicklas R, et al. ECHS1 deficiency as a cause of severe neonatal lactic acidosis. JIMD Rep. 2016;30:33-7.

8. Nair P, Hamzeh AR, Mohamed M, Malik EM, Al-Ali MT, Bastaki F. Novel ECHS1 mutation in an Emirati neonate with severe metabolic acidosis. Metab Brain Dis. 2016;31(5):1189-92.

9. Olgiati S, Skorvanek M, Quadri M, et al. Paroxysmal exercise-induced dystonia within the phenotypic spectrum of ECHS1 deficiency. Mov Disord. 2016;31(7):1041-8.

10. Mahajan A. AUID- oho, Constantinou J, Sidiropoulos C. ECHS1 deficiencyassociated paroxysmal exercise-induced dyskinesias: case presentation and initial benefit of intervention. J Neurol. 2017;264(1):185-7.

11. Bedoyan JK, Yang SP, Ferdinandusse $\mathrm{S}$, et al. Lethal neonatal case and review of primary short-chain enoyl-CoA hydratase (SCEH) deficiency associated with secondary lymphocyte pyruvate dehydrogenase complex (PDC) deficiency. Mol Genet Metab. 2017;120(4):342-9.

12. Huffnagel IC, EJW R, Reneman L, Vaz FM, Ferdinandusse S, Poll-The BT. Mitochondrial encephalopathy and transient 3-Methylglutaconic Aciduria in ECHS1 deficiency: long-term follow-up. JIMD Rep. 2018;39:83-7.

13. Al MF, Shamseldin HE, Alfadhel M, Rodenburg RJ, Alkuraya FS. A lethal neonatal phenotype of mitochondrial short-chain enoyl-CoA hydratasedeficiency. Clin Genet. 2017;91(4):629-33.

14. Balasubramaniam S, Riley LG, Bratkovic D, et al. Unique presentation of cutis laxa with Leigh-like syndrome due to ECHS1 deficiency. J Inherit Metab Dis. 2017:40(5):745-7.

15. Ogawa E, Shimura M, Fushimi T, et al. Clinical validity of biochemical and molecular analysis in diagnosing Leigh syndrome: a study of 106 Japanese patients. J Inherit Metab Dis. 2017:40(5):685-93.

16. Fitzsimons $\mathrm{PE}$, Alston $\mathrm{CL}$, et al. Clinical, biochemical, and genetic features of four patients with short-chain enoyl-CoA hydratase (ECHS1) deficiency. Am J Med Genet A. 2018;176(5):1115-27.

17. Carlston CM, Ferdinandusse S, Hobert JA, Mao R, Longo N. Extrapolation of variant phase in mitochondrial short-chain Enoyl-CoA Hydratase (ECHS1) deficiency. JIMD Rep. 2018;43:103-9.

18. Shayota BJ, Soler-Alfonso C, Bekheirnia MR, et al. Case report and novel treatment of an autosomal recessive Leigh syndrome caused by short-chain enoyl-CoA hydratase deficiency. Am J Med Genet A. 2019;179(5):803-7.

19. Alkonyi I, Sándor A. Demonstration of an enzymatic transfer reaction between beta-methyl-crotonyl-coenzyme a and L-carnitine. Acta Biochim Biophys Acad Sci Hung. 1972;7(2):149-50.

20. Sharpe AJ, McKenzie M. Mitochondrial Fatty Acid Oxidation Disorders Associated with Short-Chain Enoyl-CoA Hydratase (ECHS1) Deficiency. Cells. 2018;7(6):46.

21. Peters H, Ferdinandusse S, Ruiter JP, Wanders RJ, Boneh A, Pitt J. Metabolite studies in HIBCH and ECHS1 defects: implications for screening. Mol Genet Metab. 2015;115(4):168-73.

\section{Publisher's Note}

Springer Nature remains neutral with regard to jurisdictional claims in published maps and institutional affiliations.

Ready to submit your research? Choose BMC and benefit from:

- fast, convenient online submission

- thorough peer review by experienced researchers in your field

- rapid publication on acceptance

- support for research data, including large and complex data types

- gold Open Access which fosters wider collaboration and increased citations

- maximum visibility for your research: over $100 \mathrm{M}$ website views per year

At BMC, research is always in progress.

Learn more biomedcentral.com/submissions 